\title{
Sichere Gesundheit - gesunde Sicherheit: \\ Pandemien als objektives und subjektives (Sicherheits-)Risiko
}

\author{
Stefan Engert*
}

\begin{abstract}
The outgrowths of globalization - in particular the increase in airtravel, population growth, urbanization (mega-cities), and the advancement of the human species into the tropical rainforests - have increased the likelihood of worldwide pandemic events such as SARS or swine flu (objective insecurity). In addition, international organizations and nation states also perceive themselves more vulnerable to pandemic diseases (subjective insecurity). The twin effect has resulted in an increasing social construction of bacteria and viruses as a national security threat. The securitization of health undoubtedly entails the benefit of countering such threats more effectively, yet, is also problematic if it goes together with a substantial curtailing of human- as well as citizens rights. A case in point is Singapore's fight against SARS in 2003.
\end{abstract}

Keywords: Health policy, pandemics, security, governance, SARS, Singapore, securitization Gesundheitspolitik, Pandemien, Sicherheit, Governance, SARS, Singapur, Versicherheitlichung

\author{
„Viren sind unsere einzigen Rivalen um \\ die Herrschaft auf diesem Planeten. “ \\ Joshua Lederberg, \\ Medizin-Nobelpreisträger (1958)
}

\section{Einleitung}

I n ihren Auswirkungen unterscheiden sich Kriege und Pandemien ${ }^{1}$ kaum voneinander: Waffen und Erreger zerstören ganz oder teilweise den menschlichen Körper und stellen bei massenhaftem Auftreten die Funktionsfähigkeit und das Überleben von Staaten und Gesellschaften in Frage. Durch Seuchen sind nicht nur historisch gesehen mehr Menschen zu Tode gekommen als durch Kriege; erstere sind auch heute noch die weltweit häufigste Todesursache (Malaria, Tuberkulose, Hepatitis, etc.).

Schon im 2. und 5. Jahrhundert n. Chr. wüteten im gesamten Gebiet des Römischen Imperiums die aus dem südöstlichen Mittelmeerraum eingeschleppte Antonionische und Justitianische Pest. Um das Jahr 1350 rollte dann der Schwarze Tod, die Beulenpest, von Mittelasien aus durch Europa und forderte mehr als 25 Millionen Todesopfer - ein Drittel der damaligen Bevölkerung. Die bisher größte Pandemie war allerdings die Spanische Grippe. Die durch Tröpfcheninfektion übertragene Lungenkrankheit tötete in den Jahren 1918-20 weltweit über 50 Millionen Menschen - mehr als die Kriegshandlungen des Ersten Weltkriegs (vgl. Youngerman 2008: 3, 16, 95-98).

Diese kurze Aufzählung zeigt, dass Viren und Bakterien schon immer ein grenzüberschreitendes Phänomen waren und damit prädestiniert für die Art des internationalen Regierens sind, die man gemeinhin als „Governance“ bezeichnet, also die kooperative Steuerung gemeinsamer Interdependenzen und Problem-

* Dr. Stefan Engert ist wissenschaftlicher Mitarbeiter an der Goethe-Universität Frankfurt/Main. Der Autor dankt dem BMBF für die finanzielle Unterstützung des Forschungsprojekts. Dieser Beitrag ist anonym begutachtet worden.

1 Eine Pandemie ist eine durch Viren oder Bakterien übertragene Infektionskrankheit, die sich innerhalb kurzer Zeit mit einer Vielzahl an Krankheitsund Todesfällen über mehrere Länder oder Kontinente hinweg ausbreitet (Youngerman 2008: 5-6). lagen auf internationaler Ebene durch nicht ausschließlich intergouvernementale Akteure (Benz 2004: 15-17). Nach dem Ende des Kalten Kriegs und dem Wegfall der politisch-ideologischen Schranken des Ost-West-Konflikts wurde Gesundheitspolitik - ebenso wie viele andere Bereiche (z.B. Umwelt oder Wirtschaft) - vermehrt in den Zuständigkeitsbereich globaler Institutionen und Akteure verlagert bzw. um diese erweitert. Diese Entwicklung wird in der wissenschaftlichen Literatur unter dem Stichwort global health governance bzw. public health behandelt (vgl. Cooper et al. 2007).

Weniger offensichtlich ist demgegenüber, dass das Low-politicsThema Gesundheit in der letzten Dekade ebenso mit dem Label "Sicherheit" in Verbindung gebracht wurde, also das private bzw. persönliche Risiko „Krankheit“ als health security in den High-politics-Bereich der internationalen Politik (vgl. EU 2003; UN 2004; WHO 2007) und der Internationalen Beziehungen (Price-Smith 2002; Davies 2008; Enemark 2009; Elbe 2010a, b) aufgerückt ist. ${ }^{2}$ Die allgemeine Annahme ist, dass die Globalisierung die inhaltlichen Bezüge zwischen den Themen Gesundheit und Sicherheit verstärkt und auf die globale Agenda (zurück) gebracht hat (Elbe 2010b: 414). Die analytische Frage, die sich dieser Beitrag stellt, ist, worin genau die Gründe dafür liegen bzw. wie das Sicherheits-Upgrade von „Gesundheit“ zu erklären ist. Zwei Faktoren sind meines Erachtens für diese Entwicklung ursächlich: (1) Die Veränderungen in der objektiven (Un-)Sicherheitslage, d.h. der Anstieg des mathematischen bzw. statistischen Risikos von Pandemieereignissen, durch die Globalisierung, und (2) die Zunahme der subjektiven (Un-)Sicherheit, d.h. die Ergebnisse der Diskussions- und Aushandlungsprozesse innerhalb der (inter-)nationalen Gesellschaft, die Viren und Bakterien vermehrt als erhöhtes Sicherheitsrisiko konstruieren.

Der Artikel beginnt zunächst mit der Analyse der objektiven Sicherheitslage und zeigt mittels entsprechender Statistiken, dass das Risiko eines globalen Pandemieausbruchs durch die Globalisierung tatsächlich angestiegen ist. Danach folgt die

2 Der Artikel konzentriert sich auf Pandemien. Die Phänomene AIDS sowie der Biowaffen-Terrorismus, z.B. die Anthrax-Anschläge in den USA im September 2001, bleiben der Betrachtung außen vor. 
Analyse der subjektiven (Un-)Sicherheitslage. Diese zeigt, dass das Bedrohungsrisiko von Viren und Bakterien (inter-)national seit Mitte der 1990er Jahre verstärkt wahrgenommen und in den verschiedenen nationalen Gesellschaften als Sicherheitsproblem konstruiert wird. Zum Abschluss wechselt der Artikel noch einmal kurz die Analyseebene, indem er von der Makroauf die Mesoebene schwenkt und am Beispiel der Bekämpfung der Severe Acute Respiratory Syndrome (SARS)-Pandemie in Singapur (2003) die Auswirkungen der „Versicherheitlichung“ (Buzan et al. 1998) staatlicher Gesundheitspolitik bzw. von Pandemien am Einzelfall etwas konkreter darstellt und plausibilisiert. Abschließend werden die politischen Implikationen dieser Entwicklung im allgemeinen Rahmen von Effektivität und Legitimität diskutiert.

\section{Objektive Sicherheit: Die Rückkehr der Seuchen}

Aufgrund der technischen und medizinischen Fortschritte des 20. Jahrhunderts (Kanalisierung, Hygiene, Ernährung/ Vitaminversorgung und Impfstoffentwicklung) prognostizierte Medizin-Nobelpreisträger Frank Macfarlane Burnet in den 1970er Jahren, dass Infektionskrankheiten im 21. Jahrhundert weitgehend ausgerottet sein würden. ${ }^{3}$ Schon zehn Jahre später zerstörte AIDS bzw. das HIV-Virus diesen Mythos. ${ }^{4}$ Im Gegensatz zu den früheren, optimistischen Prognosen kehrten in den 1990er und 2000er Jahren nicht nur die „alten“ Seuchen zurück, z.B. die Cholera (Peru, 1991), die Pest (Indien, 1994) und die Diphtherie (Russland, 1995), sondern ganz „neue“ und bisher unbekannte Erreger erschienen zudem auf der Bildfläche: BSE (2001), SARS (2003) sowie die Vogel- (Influenza A/H5N1; 2004) und die Schweinegrippe (Influenza A/H1N1; 2009/10) (vgl. Youngerman 2008: 22; WHO 2007: 7, 12). Die zurzeit noch auf Norddeutschland begrenzte Epidemie durch den Darmkeim EHEC (Mai/Juni 2011) reiht sich durchaus in diese Reihe der sogenannten (re-)emerging infectious diseases ein. Innerhalb von 20 Jahren nach Ende des Kalten Kriegs haben weltweite Infektionswellen bzw. Pandemieereignisse die faktischen Rahmenbedingungen globaler Gesundheitspolitik verändert. Das Risiko eines gesamtgesellschaftlichen bzw. individuellen Schadens durch Viren und Bakterien ist heute wieder wahrscheinlicher geworden.

Was aber erklärt die unvermutete Wiederkehr der Seuchen in Zeiten der Globalisierung trotz besserer technischer Warnsysteme, effektiverer medizinischer Versorgung und gestiegener Hygiene? Es sind, wie nachfolgend gezeigt wird, drei unmittelbare Globalisierungsfaktoren, die eine erhöhte Verwundbarkeit der Menschheit gegenüber Viren und Bakterien ausgelöst haben: gestiegener Flugverkehr, Verstädterung und die Abholzung des Regenwaldes.

3 Der Spiegel, 19. April 1999.

4 Der Spiegel, Titelstory vom 6. Juni 1983.

\subsection{Globaler Reise- und Flugverkehr}

Historische Analysen zum Verlauf der Pest zeigen, dass sich Infektionskrankheiten bevorzugt über die großen Handels- und Reiserouten und über Waren- und Verkehrsdrehkreuze (damals Hafenstädte, heute Flughäfen) ausbreiteten (vgl. WHO 2007: 2-3). Die technischen Fortschritte in den Bereichen Logistik und Transport haben die Dichte der sozialen Beziehungen erhöht, das Tempo der gesellschaftlichen Austauschprozesse gesteigert und somit den Seuchen einen Geschwindigkeitsschub verschafft. Die gestiegene Mobilität ihrer „Wirte“ hat die Übertragungsgeschwindigkeit von Mikroorganismen beschleunigt und damit die allgemeine Ansteckungswahrscheinlichkeit signifikant erhöht.

Während die Pest im Mittelalter noch drei Jahre brauchte um von Süd- nach Nordeuropa vorzudringen (WHO 2007: 3), sorgt das „Jet-Zeitalter“ mit seinem weltweiten, verbilligten Reiseverkehr (vgl. IATA 2010: 10, 12) und inter-kontinentalen Flugrouten dafür, dass jeder Punkt der Erde nicht nur für Touristen und Geschäftsreisende, sondern auch für Krankheitserreger innerhalb von weniger als 36 Stunden erreichbar ist. Singapore Airlines fliegt beispielsweise heute in nur 18 Stunden nonstop von Südostasien um die halbe Welt nach New York. Die Globalisierung hat somit unintendiert ein optimales Ausbreitungsnetz für den schnellen Export von Viren über große Entfernungen geschaffen (Gottschalk 2009: 1, 5). ${ }^{5}$ Zudem hat sich die Mobilität durch gesunkene Transportkosten (Hummels 2007: 131-33, 151) erhöht und zu einem überproportionalen Anstieg des Ferntourismus von 25 Millionen Touristen im Jahr 1950 auf 922 Millionen im Jahr 2008 geführt (UNWTO 2009). Der weltweite zivile Flugverkehr nimmt jährlich um ca. fünf Prozent zu (Umweltbundesamt 2005: 2) - dies gilt für Passagierzahlen und Passagierkilometer gleichermaßen. Das heißt, dass nicht nur immer mehr Menschen reisen, sondern dies auch zu weiter entfernten Zielen. Zwei Drittel der ca. 30 Millionen Flüge im planmäßigen Linien- und Charterflugverkehr 2008 fanden im Rahmen von Urlaubsreisen statt (DLR 2009; Umweltbundesamt 2005: 13). Angesichts der heutigen kurzen Reisezeiten und der im Vergleich dazu längeren Inkubationszeiten von Infektionskrankheiten scheint die Kontrolle von Krankheitserregern und ihrer Ausbreitung mittels Fieberkontrolle und Wärmebildkameras in den Sicherheitsschleusen von Flughäfen nahezu illusorisch, da sie, bis erste Symptome auftreten, unbemerkt als „blinde Passagiere“ durch jede Grenzkontrolle kommen. Der superspreader SARS wurde so im Frühjahr 2003 in nur zwei Tagen von Hongkong aus in drei weitere Kontinente eingeschleppt (Gottschalk 2009: 3-4). 50 Prozent der weltweiten Krankheitsfälle (8.000 Infizierte, davon 918 Tote) lassen sich auf diesen ersten Kranken oder „Indexpatienten“ zurückführen. SARS hat eine maximale Inkubationszeit von zehn Tagen (WHO 2003); in diesem Zeitraum kann man sechsmal um die ganze Welt fliegen.

5 Berliner Zeitung, 17. August 1995. 


\subsection{Bevölkerungswachstum und Urbanisierung}

Ein weiterer Grund, weshalb sich die geografische Ausbreitung von Viren und Bakterien deutlich erhöht hat, ist die gestiegene Zahl der „Wirte“ (Menschen), die die Krankheiten übertragen können. Das hohe Bevölkerungswachstum hat die Zahl der potenziellen menschlichen Träger von Krankheitserregern vervielfacht; Urbanisierung und Metropolisierung haben die soziale Distanz reduziert und damit die Wahrscheinlichkeit einer Übertragung zusätzlich erhöht.

Das Wachstum der Erdbevölkerung hat seit der Industrialisierung exponentiell zugenommen: Während im Jahr 1900 nur ca. 1,7 Milliarden Menschen auf der Erde lebten, sind es heute fast sieben Milliarden.; die Bevölkerungsdichte lag 1950 noch bei durchschnittlich 19 Personen pro Quadratkilometer, heute liegt sie bei 51. ${ }^{6}$ Das heißt, dass immer weniger Menschen räumlich voneinander getrennt und stattdessen konzentrierter an wenigen Orten leben: Während im Jahr 1950 nur ca. 29 Prozent der Weltbevölkerung in Städten lebten, sind es heute schon 50 Prozent (UN/DESA 2007: 4-5). Parallel dazu hat sich die Zahl der Millionenstädte von 17 (1900) auf 429 (2009) und

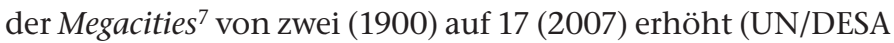
2007: 10-12). Der verstärkte Zuzug der Landbevölkerung in die Städte führt zudem zu einer „Urbanisierung der Armut“: Ein Drittel der städtischen Weltbevölkerung ist von Verslumung direkt betroffen und lebt unter Bedingungen unzureichender Hygiene, mangelnder Trinkwasserversorgung und fehlender sanitärer Einrichtungen in provisorischen Selbstbauhüttensiedlungen (UN-Habitat 2010: 9-20). Von 1975 bis 2005 hat sich die Zahl der in Slums lebenden Menschen auf rund 1 Milliarde verdoppelt. ${ }^{8}$ Die urbanen Ballungszentren mit ihrer extrem hohen Bevölkerungsdichte und teilweise mehreren Flughäfen sind daher zu optimalen Verbreitungsplattformen „mutiert“, wo Viren konzentriert an viele Wirte gleichzeitig weitergegeben werden können.

\subsection{Raubbau des Tropischen Regenwaldes}

In den Biodiversitäts-Hotspots der tropischen Urwälder - der Regenwaldgürtel liegt jeweils zehn Breitengrade unter- bzw. oberhalb des Äquators - konzentrieren sich ca. 60 Prozent aller bekannten Tier- und Pflanzenarten (Myers et al. 2000) und ca. 80 Prozent aller Mikro-Organismen. Damit sind sie das größte Erregerreservoir der Welt. Die konstant warmen Temperaturen und die hohe Luftfeuchtigkeit sorgen für optimale Lebensbedingungen der Viren, Bakterien und pathogenen Pilze. Durch die Abholzung und Brandrodung der Urwaldgebiete dringt der Mensch immer tiefer in diese bedeutende und bisher unzugängliche, unberührte ökologische Nische vor. Holzfäller, Bauarbeiter, Jäger und Wilderer kommen so immer öfter mit exotischen Tieren in Kontakt, die als Wirte und Vektoren bisher unbekannte Krankheiten vom Tier auf den Menschen übertragen können

6 United Nations: Department of Economic and Social Affairs (DESA), Population Division: World Population Prospects, The 2008 Revision, http://esa. un.org/UNPP/p2k0data.asp [10. Februar 2011].

7 Zehn Millionen und mehr Einwohner.

8 Bundeszentrale für politische Bildung 2008: Megastädte-Slums, http://www. bpb.de/themen/11375H,0,0,Slums.html [10. Februar 2011]. (vgl. Maheshwari 2001; Keesing et al. 2010). ${ }^{9}$ Dadurch hat sich das Infektionsrisiko zumindest kurz- und mittelfristig (konkav) deutlich erhöht. ${ }^{10}$

Die hoch tödlichen Viren HIV (übertragen durch Affen, Zentralafrika), Ebola (Überträger sind Fledermäuse, Kongo) und die Erreger des Dengue-Fiebers (ebenfalls übertragen durch Affen, malaiische Halbinsel) sind allesamt „Regenwald-Karrieren“. Sie werden in der Virologie als emerging infectious diseases bezeichnet, d.h. bisher unbekannte Erreger, die von Tieren auf Menschen „übergesprungen“ sind (Zoonose) (Gottschalk 2009: 1). Die Unbekanntheit bzw. Neuheit macht es für die Medizin und Virologie sehr schwierig, entsprechende Impfstoffe und Medikamente rechtzeitig entwickeln und im Bedarfsfall bereitstellen zu können.

Als Zwischenfazit dieses Teils lässt sich zunächst festhalten, dass das statistische Risiko eines Pandemieausbruchs durch die Globalisierung im Vergleich zu 1990 weltweit angestiegen ist. Die meisten Mikrobiologen, wie z.B. der Niederländer Jan Osterhaus, kommen daher zu dem Schluss, dass der Menschheit die gefährlichsten Seuchen in ihrer Geschichte erst noch bevorstehen. ${ }^{11}$

\section{Subjektive Unsicherheit: Die konstruierte Bedrohung durch Pandemien}

Ob man Krankheiten als Gesundheits-, Entwicklungs-, Wohlfahrts- oder als Sicherheitsproblem definiert und ob man das Ausmaß der Risikobetroffenheit als individuell oder kollektiv versteht und damit Pandemien eher mit medizinischen Mitteln oder einer militärnahen Vorgehensweise „bekämpft“ ist vor allem eine politische Entscheidung, die von der Risikowahrnehmung und -beurteilung der jeweiligen Gesellschaft und ihrer Regierung abhängt. Gefährdungen werden erst dann zu gesellschaftlich relevanten Sicherheitsrisiken, wenn sich nicht nur ihre Eintrittswahrscheinlichkeit objektiv-mathematisch berechnen lässt, sondern wenn diese auch durch soziale Kommunikationsprozesse als solche identifiziert bzw. konstruiert werden. Sicherheitskultur ist daher immer ein gegenseitiges Wechselspiel zwischen der objektiven Risikolage und der subjektiven (Un-)Sicherheit zu verstehen. Allerdings sind Risiken nicht nur rein kulturell geprägte Institutionen, da subjektive Sicherheit nun mal nicht unabhängig von den realen Gefahren gedacht werden kann. Andererseits werden das „tatsächliche“ Risiko bzw. bestimmte Themen und Inhalte erst durch gesellschaftliche Kommunikationsprozesse politisch relevant und damit ver- oder entsicherheitlicht. Was davon Ursache und was Wirkung ist - die materiellen Sachzwänge oder der politische Interpretationsrahmen -, ist eine empirisch letztendlich nur schwer zu beantwortende Frage.

Seit den 1990er Jahren hat das gesellschaftliche Bewusstsein gegenüber globalen Risiken generell wie auch die Wahrnehmung der eigenen Betroffenheit durch Viren und Bakterien zugenommen. Das Internet, dessen Verbreitung unaufhörlich

\footnotetext{
9 Vgl. Der Spiegel, 5. Januar 2009, 19. April 1999 und 27 April 1992.

10 Die Welt, 2. Mai 1996; Der Spiegel, 23. Juni 2003.
}

11 Rhein-Neckar-Zeitung, 23. Februar 2007. 
voranschreitet, ${ }^{12}$ sorgt an dieser Stelle nicht nur für eine Beschleunigung der globalen Kommunikation, sondern auch für eine erhöhte individuelle Betroffenheitswahrnehmung. Der alltägliche Zugriff auf die Medien-, Nachrichten- und Netzwerkangebote des Internets hat eine globale Vernetzung der Nutzer zur Folge, die sich immer mehr zu einer virtuellen „Weltrisikogemeinschaft“ (Beck 2008) auswächst. Der Mikroblog Twitter ermöglicht mittlerweile sogar eine Echtzeitkommunikation von Risiken. Gerade bei Pandemien lässt sich dieser vorlaufende Informations- und Kommunikationseffekt gut beobachten, wenn etwa die Panik vor einer Seuche schon um sich greift, noch bevor die Krankheit tatsächlich die nationalen Grenzen überschritten hat (Briseno 2009). In Zeiten der Globalisierung bestimmt daher die rein technisch-objektive Gefahrenabschätzung nicht mehr ausschließlich den Grad der (Un-)Sicherheit einer Gesellschaft - die subjektive Sicherheit ist genauso entscheidend (vgl. Gusy 2010: 112-113).

Am Beispiel der Infektionskrankheiten lässt sich sehr gut zeigen, dass sich global sowohl die Einstellungen als auch das Verhalten der betroffenen Akteure (Staaten) verändert haben: In den Internationalen Beziehungen hat zum einen die Erweiterung der Sachdimension des Sicherheitsbegriffs (Daase 2010: 4-9) dazu geführt, dass das Thema öffentliche Gesundheit - ebenso wie die Themen Energiesicherheit und cyber security - in der 2000er Dekade in den Bereich der das staatliche Überleben existenziell gefährdender Bedrohungen hineindefiniert wurden. ${ }^{13}$ Zum anderen hat die Erweiterung der Referenzdimension des Sicherheitsbegriffs (Daase 2010: 9-12) um das Konzept der human security ${ }^{14}$ dazu geführt, dass private Risiken (wie z.B. Krankheit) vermehrt in den Deutungsrahmen eines öffentlichen und „entgrenzten“ Risikos für die jeweilige Gesellschaft und sogar die Menschheit als Ganzes gerückt wurden (vgl. Koblentz 2010: 99-100). Viren und Bakterien werden damit nicht mehr nur als eine persönliche bzw. individuelle Angelegenheit des betroffenen Kranken, sondern als eine kollektive Existenzbedrohung für alle Staaten und ihre Bürger wahrgenommen.

Diese beiden Aspekte bilden für viele Staaten die argumentative Basis, die konkrete Gefahrenabwehr von Viren und ihrer Wirte im „Notfall“ den Standardabläufen des politischen Systems entziehen zu können, um nachhaltige Schäden durch temporäre Grenzschließungen, Reise-, Handels- und Tourismuseinschränkungen verhindern zu können (Koblentz 2010: 121-122). Wenn Pandemien als nationale Sicherheitsbedrohung identifiziert bzw. „versicherheitlicht“ werden, legitimiert die Notwendigkeit der effektiven Gefahrenabwehr in der konkreten Ausnahmesituation auch den Rückgriff auf außergewöhnliche Sicherheitsmaßnahmen (vgl. UN 2004: 30) bzw. Durchführungsbestimmungen, die mit einer Einschränkung oder Aussetzung individueller Freiheits-, Bürger- und Menschenrechte einhergehen können.

12 Waren 1988 lediglich acht Staaten mit dem Internet verbunden, ist es heute jeder Staat.

13 Der ursprünglich rein militärisch definierte Sicherheitsbegriff hatte sich schon durch die Integration der Low-politics-Bereiche Wirtschaft (1970er), Umwelt (1980er) und humanitärer Bedrohungen (1990er) vorher deutlich verbreitert.

14 Human security fokussiert auf das Individuum als Bezugspunkt von Sicherheit (vgl. Suhrke 1999).
Wie der nachfolgende Abschnitt zeigt, ist das mathematisch bzw. materiell angestiegene Risiko auch in der weltgesellschaftlichen Wahrnehmung von Unsicherheit allgegenwärtig. Die Risikosituation betreffend Pandemien wird auf der globalen Ebene seit der Jahrtausendwende unisono als existenzielle Bedrohung aufgefasst. Den Annahmen der Kopenhagener Schule folgend, lassen sich die nachfolgenden Sprechakte gouvernementaler Akteure klar als „securitization moves“ klassifizieren (Buzan et al. 1998:23-29):

Im Jahr 2000 hat der UN-Sicherheitsrat zum ersten Mal ein Gesundheitsthema unter dem Tagesordnungspunkt Sicherheit diskutiert: „The impact of AIDS on peace and security in Africa“. ${ }^{15}$ In ihrem Strategiepapier zur zukünftigen Sicherheit vier Jahre später identifiziert die UN insgesamt sechs „major threats to international peace and security", wozu sie auch neue Infektionskrankheiten zählt (vgl. UN 2004: xi, 25, 29-30):

"The security of the most affluent state can be held hostage to the ability of the poorest State to contain an emerging disease. [...] Any one of 700 million international airline passengers every year can be an unwitting global disease-carrier [...] The influenza pandemic of 1919 killed as many as 100 million people, [...] Today, a similar virus could kill tens of millions in a fraction of the time." (UN 2004: 14)

Im Weltgesundheitsbericht der WHO von 2007 wird sogar schon im Untertitel der Bezug zwischen „Gesundheit” und "Sicherheit" hergestellt: Anstelle von health governance oder global health policy wird gleich von "global public health security" (WHO 2007) gesprochen.

Die nationalstaatlichen Diskurse liegen nicht anders und zeugen ebenfalls von einem breiten Gebrauch versicherheitlichender Vokabeln: Die aktuelle National Security Strategy der USA weist Gesundheitsbedrohungen wie Pandemien einen substanziellen Sicherheitsstatus zu: Infektionskrankheiten werden hier als eine "key global challenge [...] threatening the security of regions and the health and safety of the American people" (Potus 2010: 8, 48-49) definiert. Schon zehn Jahre zuvor hatte Washington zum ersten Mal die Infektionskrankheit AIDS als eine Bedrohung für die nationale Sicherheit und den Frieden ausgemacht (vgl. Koblentz 2010: 96). Die Risikoanalyse der britischen Regierung steht diesen Formulierungen in nichts nach:

"The Cold War threat has been replaced by a diverse but interconnected set of threats and risks, which affect the United Kingdom directly and also have the potential to undermine wider international stability: They include [...] pandemics." (UK 2008: $3,14-15)$

Die konkrete Bekämpfung von Pandemien durch die Staatenwelt ist jedoch nicht nur ein Lippenbekenntnis; die Bedrohungswahrnehmung wird zunehmend auch im Verhalten „versicherheitlicht“, d.h. in entsprechende Handlungsanleitungen umgesetzt: So arbeitet gleich eine Reihe von internationalen Krisenstäben wie z.B. das WHO Global Outbreak Alert and Response Network (GOARN) (UN 2004: 29-30) an der pro-aktiven, fast militärischen Bekämpfung von Pandemien. Für den kon-

15 Bundeszentrale für politische Bildung 2010: Global Health Governance, http://www.bpb.de/files/MY3P0C.pdf [15. Februar 2011]. 
kreten Krisenfall wurden nationale Pandemiepläne entwickelt, die durch Gesundheitsbürokratien wie etwa das Robert Koch-Institut (RKI) in Berlin, das Center for Disease Control and Prevention in Atlanta (USA) und die Word Health Organization (WHO) in Genf autoritativ verwaltet werden. Die WHO selbst verfügt über einen sechsstufigen Pandemien-Vorwarnplan (WHO 2005: 2), der dem militärischen Alarmzustandsplan der US-amerikanischen Verteidigungsbereitschaft (DEFCON) sehr ähnlich ist. Am 11. Juni 2009 hat die WHO wegen der drohenden Schweinegrippe-Pandemie erstmals die höchste Warnstufe ausgerufen. ${ }^{16}$ Das RKI prognostizierte damals für Deutschland eine hohe Zahl an Toten und einen volkswirtschaftlichen Schaden durch „Produktionsausfälle von bis zu 45 Milliarden Euro“, ${ }^{17}$ zu denen es aber bekannterweise nie gekommen ist. Die deutschen Bundesländer blieben auf ungenutzten Impfdosen im Wert von 283 Millionen Euro sitzen ${ }^{18}$ und die WHO musste sogar Gerüchte einer „fake pandemic“ dementieren. ${ }^{19}$

Ein kurzer Blickwechsel auf die individualstaatliche Ebene verdeutlicht, dass es Gesellschaften gibt, die wesentlich „versicherheitlichter" bzw. militärischer auf eine Gesundheitsbedrohung reagiert haben als die Bundesrepublik im Jahr 2010: So hat bspw. die Staatsführung Singapurs im Frühjahr 2003 ganz offiziell den „Krieg gegen SARS“ ausgerufen (Weber et al. 2008: 147; vgl. 154-7; Hudson 2008). Am 24. März 2003 verabschiedete das nationale Ministerium für Gesundheit den sogenannten „Infectious Diseases Act“. Dieser beinhaltete Notstandserlasse, die partiell an martial law light erinnern. Im Anschluss an den sprachlichen bzw. legalen Versicherheitlichungsakt hat die Regierung des Stadtstaats mit teilweise militärisch anmutenden Mitteln versucht, die Pandemie einzudämmen.

Zunächst wurden alle diagnostizierten SARS-Patienten zentral im Tan-Tock-Seng-Krankenhaus in einer 21 Tage andauernden Zwangsquarantäne isoliert, ohne dass eine Besuchsmöglichkeit für Angehörige oder Freunde bestand. Alle Verdachtsfälle - also diejenigen Personen, die mit SARS-Patienten in Kontakt kamen - wurden zudem vorsorglich in eine 14 Tage andauernde Heimquarantäne beordert (vgl. Kamps/Hoffmann 2003: 85, 89). Die Einhaltung der Quarantäne wurde durch zufällige Kontrollanrufe und mittels einer permanenten elektronischen Kamera-Überwachung der Eingangstür durch die halb-private Sicherheitsfirma CISCO, einer Art Hilfspolizei, kontrolliert (vgl. Hudson 2008: 171). Verdächtige, die ihre Heimquarantäne nicht einhielten oder dreimal telefonisch nicht erreichbar waren, bekamen elektronische Fußfesseln angelegt. ${ }^{20}$ Wer gegenüber dem befragenden Arzt falsche Angaben machte oder die Quarantäne durchbrach, konnte direkt und ohne ordentliches Gerichtsverfahren mit einem Bußgeld von ca. 2.500 Euro oder mit bis zu einem Jahr Freiheitsentzug belegt werden. ${ }^{21}$ Zudem wurden alle Quarantänebrecher in der Straits Times, Singapurs größter Tageszeitung, mit Namen und Foto veröffentlicht und als „rücksichtslos“ bzw. „Gefahr“ verfemt. ${ }^{22}$ Darüber hinaus

\footnotetext{
16 http://www.who.int/mediacentre/news/statements/2009/h1n1_pandemic phase6 20090611/en/index.html [2. Februar 2011].

17 Der Spiegel, 8. März 2010.

18 Spiegel Online, 7. Mai und 11. August 2010.

19 http://www.who.int/mediacentre/news/statements/2010/h1n1_pandemic_ 20100122/en/index.html [2. Februar 2011].

20 FAZ, 25. April 2003.

21 FAZ, 25. April 2003.

22 FAZ, 24. April 2003.
}

schloss Singapur seine Grenzen und kontrollierte jeden Einund Ausreisenden auf dem nationalen Flughafen ungefragt mittels Temperaturscannern und Wärmebildkameras auf Fieber und erteilte im Bedarfsfall entsprechende Ein- oder Ausreiseverbote (vgl. Hudson 2008: 171). ${ }^{23}$ Von 283 festgestellten Infektionsfällen starben letztendlich „nur“ 33; demgegenüber wurde „virtually every person with symptoms that might indicate SARS“ (Kamps/Hoffmann 2003: 87) - insgesamt über 2.400 Personen ${ }^{24}$ - in Zwangsquarantäne gesteckt oder unter Hausarrest gestellt. Die WHO beschrieb die „Eindämmungsbemühungen“ als „aggressiv“; das Auswärtige Amt hingegen sprach von „entschlossenen Quarantänemaßnahmen“. ${ }^{25}$ Wer nun entgegnet, dass Singapurs parlamentarisches System ja auch einige autoritäre Züge enthält, ${ }^{26}$ sei daran erinnert, dass das eindeutig demokratischere Kanada im Falle von SARS ebenfalls massive Bußgelder erlassen und eine Zwangsisolierung für Quarantänebrecher verhängt hatte - wenn auch nur nach richterlichem Beschluss. Zudem hatte auch die US-amerikanische Universität Berkeley allen 500 Studenten aus China, Taiwan und Singapur, die im Sommersemester 2003 in Kalifornien ihr Studium aufnehmen wollten, vorsorglich die Einschreibung verweigert. ${ }^{27}$ Diese Beispiele zeigen, dass die „Versicherheitlichung "von Gesundheitspolitik einerseits eine vielleicht effektivere Bekämpfung von Seuchen ermöglicht, andererseits aber durchaus von einem ethischen Standpunkt aus gesehen als problematisch betrachtet werden kann und eventuell hohe Legitimitätskosten verursacht.

\section{Abschließende Bemerkungen}

Der Artikel hat versucht nachzuzeichnen, weshalb sich Gesundheitspolitik bzw. Pandemien nicht nur zu einer globalen Governance- sondern auch zu einer Sicherheitsthematik entwickelt haben. Zwei Gründen wurden dafür als ursächlich identifiziert: Einerseits die angestiegene stochastische Wahrscheinlichkeit von Pandemieereignissen seit dem Ende des Kalten Kriegs durch die Globalisierung (objektive Unsicherheit). Als Ursachen hierfür sind zu nennen der gestiegene Flugverkehr, die Urbanisierung, das allgemeine Bevölkerungswachstum und das immer tiefere Vordringen der Menschen in den Regenwald. Der vermehrte Zugang zu Viren und Bakterien, die Erhöhung der Umlaufgeschwindigkeit und die größere Anzahl an potenziellen Wirten haben so eine erhöhte Verwundbarkeit der Menschheit gegenüber Erregern zur Folge. Als zweiter Grund kann aber auch genannt werden, dass das Bewusstsein von Gesellschaften und Staaten gegenüber Risiken größer geworden ist (subjektive Unsicherheit). Neue Informationstechnologien wie das Internet haben nicht nur für eine verbilligte und schnellere Kommunikation gesorgt, sondern auch zu einem schnelleren Austausch von Informationen und Einschätzungen über Risiken und Unglücksfälle - mittlerweile sogar in Echtzeit. In Zeiten der Globalisierung schätzen Menschen die Gefahr, von

23 FAZ, 18. Mai 2003.

24 FAZ, 24. April 2003.

25 FAZ Sonntagszeitung, 18. Mai 2003.

26 Die Freedom-House-Werte von Singapur (2010) sind 5 (politische Rechte) und 4 (zivile Freiheiten) und damit gerade so der „Unfrei“-Kategorie der Diktaturen entwachsen (vgl. Hudson 2008: 167, 169).

27 Faz.net, 6. Mai 2003. 
einem globalen Risiko tatsächlich einmal selbst betroffen zu sein - ganz unabhängig von der tatsächlichen mathematischen Wahrscheinlichkeit -, vermehrt höher ein. Die Analyse des globalen Regierungsdiskurses zur Risikosituation „Gesundheit" lässt jedenfalls darauf schließen, dass Pandemien auf der inter-gouvernementalen Ebene durchaus als existenzielle Bedrohung aufgefasst und dementsprechend „versicherheitlicht“ werden.

Dieser allgemeine Befund hat sich besonders gut am Beispiel Singapurs 2003 aufzeigen lassen, das teilweise mit militärischen Mitteln auf die SARS-Pandemie reagiert hat. Im Wechselspiel zwischen subjektivem Unsicherheitsgefühl und objektiver Risikolage greifen Staaten v.a. bei akuter Betroffenheit zu außergewöhnlichen Maßnahmen, um Pandemien einzudämmen. Die Gefahr scheint dabei groß, dass ein Regierungshandeln, das auf eine möglichst effektive Problemlösung fokussiert ist, auf Kosten der individuellen Freiheits- und Bürgerrechte einer Gesellschaft geht und somit das „Kind mit dem Bade ausschüttet“. Wenn man glaubt, ein bestimmtes Maß an kollektiver Sicherheit (die Freiheit vom Virus) nur durch ein unbestimmtes Maß an individueller Unfreiheit (die Freiheit von den „Wirten“) und sozialer Ausgrenzungsmaßnahmen wie Quarantäne und Isolation „sicherstellen“ zu können, macht man, um eine weitere Metapher zu bemühen, „den Bock zum Gärtner“. In Deutschland steht die gesellschaftliche Diskussion dieser Implikationen noch aus. Die normative Herausforderung wird dabei sein, eine „sichere“ Gesundheit mit einem „gesunden“ Maß an Sicherheit zu erreichen - und nicht umgekehrt.

\section{Bibliographie}

Beck, Ulrich 2008: Weltrisikogesellschaft, Frankfurt/Main: Suhrkamp.

Benz, Arthur (Hrsg.) 2004: Governance - Regieren in komplexen Regelungssystemen, Wiesbaden: Leske\&Budrich.

Briseno, Cynthia 2009: Warum Viren und Bakterien so mächtig sind, in Spiegel Online, 31. Dezember 2009, http://www.spiegel. de/wissenschaft/medizin/0,1518,668144-2,00.html [3. Januar 2010].

Buzan, Barry/Wæver, Ole/de Wild, Jaap 1998: Security: A new framework for analysis, Boulder: Lynne Rienner.

Cooper, Andrew F./Kirton, John H./Schrecker, Ted 2007: Governing Global Health: Challenge Response Innovation, Aldershot: Ashgate.

Daase, Christopher 2010: Der erweiterte Sicherheitsbegriff, Sicherheitskultur im Wandel Working Paper Nr. 1, Frankfurt, http://www.sicherheitskultur.org/WorkingPapers/01-Daase. pdf [2. Januar 2011].

Davies, Sarah 2008: Securitizing infectious disease, in: International Affairs 84: 2, 295-313.

Deutsches Zentrum für Luft- und Raumfahrt (DLR) 2009: Global Aviation Monitor. Analysis and Short Term Outlook of Global, European and German Air Transport, March 2009, http://www.
dlr.de/Portaldata/1/Resources/portal_news/newsarchiv2009_ 2/DLR_GAM_060409_engl.pdf [3. Januar 2010].

Elbe, Stefan 2010a: Security and Global Health: Toward the Medicalization of Insecurity, Cambridge: Polity Press.

Elbe, Stefan 2010b: Health and Security, in: Collins, Alan (Hrsg.): Contemporary Security Studies, 2nd edition, Oxford: Oxford University Press, 413-427.

Enemark, Christian 2009: Is Pandemic Flu a Security Threat, in: Survival 51: 1, 191-214.

Gottschalk, René 2009: Der globale Weltverkehr und die Ausbreitung von hochinfektiösen Erregern, in: Analytik-News 25. März 2009, http://www.analytik-news.de/Fachartikel/Volltext/ Gottschalk.pdf [28. Oktober 2010].

Gusy, Christoph 2010: Sicherheitskultur - Sicherheitspolitik - Sicherheitsrecht, in: Kritische Vierteljahresschrift für Gesetzgebung und Rechtswissenschaft 93: 2, 111-128.

Hudson, Chris 2008: Singapore at War: SARS and its metaphors, in: Powers, John H. / Xiao, Xiaosui (Hrsg.): The Social Construction of SARS, Amsterdam: John Benjamins, 163-179.

Hummels, David 2007: Transportation Costs and International Trade in the Second Era of Globalization, in: Journal of Economic Perspectives 21: 3, 131-154.

International Air Transport Association (IATA) 2010: Annual Report 2010, Berlin, June 2010, http://www.iata.org/pressroom/ Documents/IATAAnnualReport2010.pdf [2. Februar 2011].

Kamps, Bernd S./Hoffmann, Christian 2003: SARS Reference - 10/2003, http://www.sarsreference.com/sarsreference.pdf [2. Februar 2011].

Keesing, F./Belden, LK/Daszak, P/Dobson, A/Harvell, CD/Holt, RD/Hudson, P/Jolles, A/Jones, KE/Mitchell, CE/Myers, SS/Bogtich, T/Ostfeld, RS 2010: Impacts of biodiversity on the emergence and transmission of infectious diseases, in: Nature 468: 647-652.

Koblentz, Gregory D. 2010: Biosecurity Reconsidered: Calibrating Biological Threats and Responses, in: International Security 34: 4, 96-132.

Maheshwari, J. K. 2001: Origin of AIDS Virus Linked to Rainforest Destruction, in: EnvioNews 7: 4 (October 2001), http:// isebindia.com/01_04/01-10-1.html [2. Februar 2011].

Myers N./Mittermeier R.A./Mittermeier C. G./Da Fonseca G. A. B./Kent J. 2000. Biodiversity hotspots for conservation priorities, in: Nature 403: 853-858.

President of the United States of America (Potus) 2010: National Security Strategy, May 2010, The White House, Washington DC, http://www.whitehouse.gov/sites/default/files/rss_viewer/ national_security_strategy.pdf [7. Januar 2011].

Price-Smith, Andrew 2002: The Health of the Nations: Infectious Disease, Environmental Change, and Their Effects on National Security and Development, Cambridge, Mass.: MIT Press. 
Suhrke, Astrid 1999: Human Security and the Interests of States, in: Security Dialogue 30: 3, 265-276.

Umweltbundesamt 2005: Entwicklung des Flugverkehrs, http:// www.umweltdaten.de/verkehr/downloads/entwflug.pdf [29. Januar 2011].

United Kingdom 2008: The National Security Strategy of the United Kingdom. Security in an interdependent world, Cabinet Office, London, March 2008, Cm 7291, http://interactive.cabinetoffice.gov.uk/documents/security/national_security_strategy.pdf [21. Januar 2011].

United Nations 2004: A more secure world: Our shared responsibility, http://www.un.org/secureworld/report2.pdf [10 Dezember 2010].

United Nations Development Programme (UNDP) 1994: Human Development Report 1994: New dimensions of human security, New York/Oxford: Oxford University Press.

UN Habitat 2010: The Challenge of Slums: Global Report on Human Settlements 2003. Revised and updated version (April 2010), http://www.unhabitat.org/downloads/docs/GRHS_ 2003_Chapter_01_Revised_2010.pdf, [29. Januar 2011]. bat SARS, in: Powers, John H. / Xiao, Xiaosui (Hrsg.): The Social Construction of SARS, Amsterdam: John Benjamins, 145-162.

World Health Organization (WHO) 2003: Global Alert and Response, Update 49 - SARS case fatality ratio, incubation period, 7 May 2003, http://www.who.int/csr/sarsarchive/2003_05_ 07a/en/ [4. Januar 2011].

World Health Organization (WHO) 2005: WHO global influenza preparedness plan, http://www.who.int/csr/resources/ publications/influenza/WHO_CDS_CSR_GIP_2005_5.pdf [29. Januar 2011].

World Health Organization (WHO) 2007: The world health report 2007: A safer future. Global public health security in the 21st century, Geneva, http://www.who.int/whr/2007/whr07_ en.pdf [4. Januar 2011].

World Tourism Organization (UNWTO) 2009: Tourism Highlights 2009 edition, http://www.hhp.ufl.edu/trsm/ctrd.default/activity/marketing_files/2009TourismHighlights.pdf [2. Januar 20011].

Youngerman, Barry 2008: Pandemics and Global Health, New York: Infobase Publishing.

\title{
Wandelt sich so Sicherheitskultur?
}

\section{Versicherheitlichungsdynamiken und Sicherheitsmaßnahmen am Beispiel des zivilen Luftverkehrs}

\author{
Susanne Fischer und Carlo Masala*
}

\begin{abstract}
The perception of what constitutes a security threat has changed during the last decade. Today, so-called new security threats such as terrorism, global crime or piracy dominate the agenda of international politics. With their transboundary character those threats affect international and national security politics. This paper investigates the consequences of these changes for national security politics, analysing securitization dynamics in the field of aviation security. Two findings stand out: First, securitization is negotiated between the securitizing actor and the respective audience. Second, national security politics transforms into what we describe as risk politics.
\end{abstract}

Keywords: Terrorism, security measures, security culture Terrorismus, Sicherheitsmaßnahmen, Sicherheitskultur

\section{Einleitung}

A m 17. November 2010 trat der damalige deutsche Innenminister Thomas de Maizière vor die Presse, um bekannt zu geben, dass sich die Gefährdungslage durch

\footnotetext{
* Susanne Fischer, M.A., ist wissenschaftliche Mitarbeiterin an der Universität der Bundeswehr München, Prof. Dr. Carlo Masala ist Professor für Internationale Politik an der Universität der Bundeswehr München. Der Beitrag entstand im Rahmen des vom Bundesministerium für Bildung und Forschung geförderten Projektes Sicherheit im öffentlichen Raum. Die Autoren danken den anonymen Gutachtern und den Mitgliedern des IB-Kolloquiums der Universität der Bundeswehr München für die hilfreichen Kommentare sowie Katrin Wagner, Jan Zöckler und - für die Unterstützung im Forschungsprozess - Franziska Heratsch.
}

den internationalen Terrorismus erhöht habe. Hintergrund waren Erkenntnisse der Sicherheitsbehörden über einen möglichen Anschlag in Deutschland. ${ }^{1}$ Die Entscheidung des Ministers, die Öffentlichkeit auf derartige sicherheitspolitische Entwicklungen hinzuweisen, löste in den deutschen Medien Diskussionen darüber aus, welcher Logik diese Äußerungen folgen, sowie darüber, was derartige offizielle Terrorwarnungen bewirken. Überdies wurde diskutiert, ob solche Warnungen wirklich geeignet sind, die Bevölkerung über eine Bedrohung der öffentlichen Sicherheit aufzuklären und Akzeptanz für

1 Bundesministerium des Inneren 2010c. 